\title{
Reflexión sobre la conservación del arte contemporáneo y su aportación a la historia del arte
}

\author{
Alicia García González
}

Resumen: El análisis histórico del arte contemporáneo plantea diversos problemas que el arte de otras épocas no presenta, y obliga a los profesionales a aplicar en muchas ocasiones una metodología de trabajo interdisciplinar. En este sentido, la labor de conservación, en la que se incluye la coordinación y asesoramiento en diferentes aspectos de la obra de arte, además de la recopilación de información y documentación, se ha convertido en una fuente fundamental para el estudio del arte. Esta labor se viene realizando de manera exhaustiva en los museos de arte contemporáneo gracias a su compromiso con la investigación, divulgación y conservación de su colección.

Palabras clave: Arte contemporáneo, conservación, restauración, investigación, documentación accesible, obra de arte.

Abstract: The research of contemporary art raises several issues that the art of the past doesn't pose. Furthermore it forces to implement an interdisciplinary methodology. In this sense, the conservation work, including coordination and advice on different aspects of the artwork, in addition to gathering information and documentation has become a key source for the study of art. This work is being carried out in the museums of contemporary art through its commitment to research, diffusion and preservation of its collection.

Keywords: Contemporary art, conservation, restoration, research, available documentation, artwork.

Resumo: A análise histórica da arte contemporânea propõe diversos problemas que a arte de outras épocas não apresenta, e obriga aos profissionais à aplicar em muitas situações uma metodologia de trabalho inter-disciplinar. Neste sentido, a labor de conservação, na qual se inclui a coordinação e assessoramento em diferentes aspectos da obra de arte, além da recopilação da informação e documentação, converteu-se em uma fonte fundamental para o estudo da arte. Esta labor vêm-se realizando de maneira exaustiva nos museus de arte contemporâneo graças ao seu compromisso com a investigação, divulgação e conservação da sua colecção.

Palavras-chave: Arte contemporâneo, conservação, restauração, investigação, documentação accessível, obra de arte.

\section{Problemas para el estudio del arte contemporáneo}

Además de la evidente falta de distancia histórica, necesaria para abordar de manera crítica el estudio del arte contemporáneo, los historiadores se encuentran con otras dificultades a la hora de crear barreras y categorías, analizar sus fuentes, influencias y consecuencias, redefinir aspectos que el tiempo obliga a cambiar, etc. Todo esto es debido a la vertiginosa velocidad con la que el arte contemporáneo se retroalimenta, se redefine, expande, se modifica en sí mismo, e incluso se autodestruye, como clara consecuencia del momento histórico en el que se desarrolla.

La diversidad de formatos y nuevas vías artísticas que surgen en el arte contemporáneo obligan a crear nuevos sistemas de documentación, catalogación y estudio. Por ello se crean y actualizan las categorías artísticas, estudiando su evolución e interrelación, analizando nuevas temáticas y su vinculación con la sociedad del momento, cuestionándose a su vez el sentido mismo del arte actual, sus bases teóricas y la validez de las barreras cronológicas, temáticas o formales que siguen una 
evolución lineal. Nuevos lenguajes artísticos aparecen con gran velocidad y se interrelacionan con otros lenguajes y soportes, el arte, la ciencia y la tecnología conviven en muchas ocasiones.

Dentro de estas nuevas vías necesarias de documentación, catalogación y estudio, el restaurador o conservador toma un papel importante. Su labor genera documentación e información sobre el artista y su obra extraordinariamente valiosa para la historia del arte.

La conservación del arte contemporáneo debe abrirse a nuevas vías de actuación, incluyendo la coordinación y asesoramiento en los desafíos que muchas veces plantean las obras de arte o las necesidades del artista, colaborando directamente con ellos en la creación o materialización de sus proyectos artísticos, además de la recopilación de información y documentación.

En la labor de conservación influyen ciertas características propias del arte contemporáneo que obligan a desarrollar protocolos y metodologías concretas. Estas características están relacionadas con los siguientes aspectos:

- La gran versatilidad de materiales y formatos.

- Las barreras entre lo material e inmaterial.

- La aparición de nuevas manifestaciones artísticas: arte efímero, arte en la calle, performance, instalaciones, video-arte, arte sonoro, net-art...

- La participación del artista en vida.

- La evolución y redefinición de la obra a lo largo del tiempo.

\section{Características de las obras de arte en los museos. Su influencia en la conservación}

Los museos de arte contemporáneo tienen la responsabilidad de estudiar, divulgar y conservar su colección. Hoy en día se han convertido en un foco generador de arte y una aportación fundamental para su estudio. Además, crean situaciones determinadas que vinculan, de un modo especial, las obras de arte con la actividad y funcionamiento del museo.

Como consecuencia, se generan proyectos artísticos nuevos, situaciones expositivas diferentes, y se realizan una serie de actividades relacionadas con su función en la sociedad, que plantean nuevos desafíos para la conservación.

Los departamentos de conservación y curatoriales establecen conjuntamente una metodología de investigación muy amplia y rigurosa de las obras, definiendo además sus competencias. Esta no se limita a la colección del museo, sino que también se establecen los mismos criterios para obras temporales, que se exponen en un momento determinado.

Para poder desarrollar esta labor, es necesario establecer diferencias y analizar en profundidad cada obra y su contexto en el museo.

De esta manera, se establecen ciertas tipologías generales:

A) Obras de arte ideadas para ser exhibidas en un espacio concreto

En muchas ocasiones los museos adquieren o exhiben obras que el artista acaba de concebir como idea, en ocasiones ex profeso para una determinada sala o exposición y para un determinado momento. El estudio de esa obra deberá ir ligado al objetivo y condiciones en las que fue creada o concebida.

La obra Escultura del artista Evaristo Bellotti fue concebida para ser expuesta en un espacio concreto, el Palacio de Cristal del Parque del Retiro en Madrid. Como parte del trabajo de 
conservación, se realizó una entrevista grabada al artista, que aportó información sobre su obra en cuanto a montaje, concepción, conservación, exposición, etc.
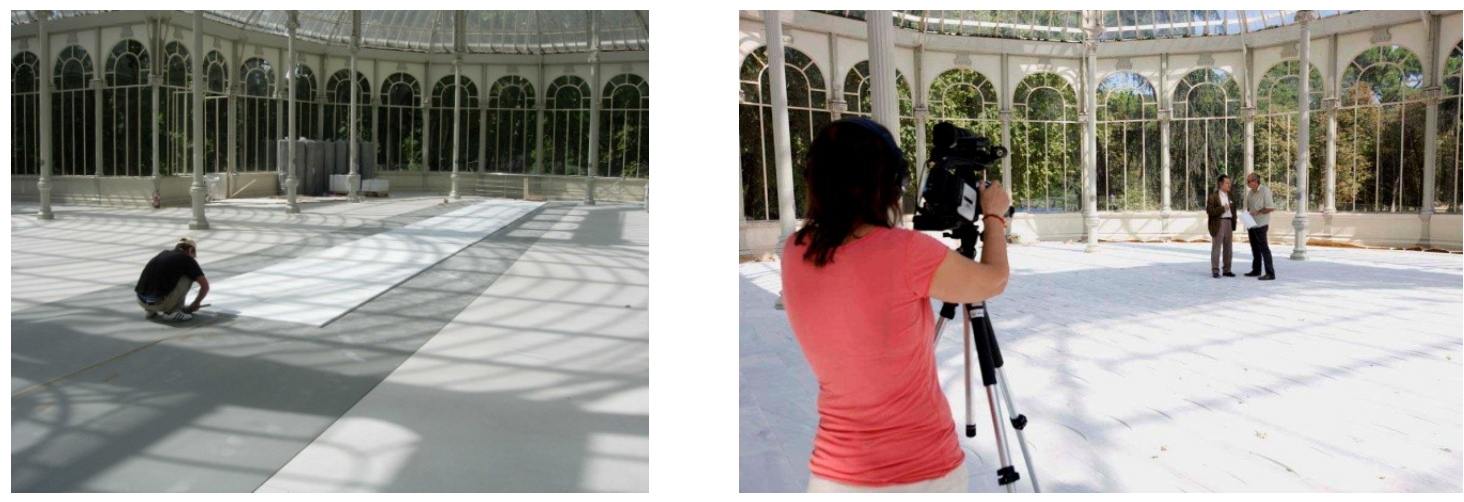

Figuras 1 y 2. Entrevista con el artista Evaristo Bellotti durante el montaje de su obra

Esto implica un trabajo interdisciplinar, ya que, en muchas ocasiones, la materialización de dicha obra supone poner en práctica una metodología de conservación y asesoramiento al artista, lo que hace que el conservador se involucre directamente en la realización y montaje de la obra, definiendo ciertos aspectos que influirán en el futuro, bien sea de manera temporal o permanente, pero de cualquier manera definitiva para el estudio de la obra y del artista. Estos aspectos son:

- El entorno de la obra o espacio expositivo.

- El medio de transmisión.

- La percepción de la obra.

- La intención del artista en cada momento determinado.

B) Obras de arte que el museo materializa y adapta a su espacio

En ocasiones, los artistas trabajan sus obras previamente como proyecto o idea inicial, que debe completarse para ser mostrada ante el público.

Algunas obras pertenecientes al denominado net-art o arte en la red, solo pueden contemplarse en un museo si se activan los procesos generadores de datos en la red, creando un espacio y medio de transmisión en cada ocasión. En otros casos la fase de interacción en la red se ha completado, pero la obra sigue desarrollándose en otros soportes, por ello es fundamental establecer la fase de creación en la que se encuentra la obra, que debe ser completada o desarrollada.

Por otro lado, también encontramos obras que solo se generan o se completan como obra de arte en contacto con el público que interactúa con ellas. En estos casos la labor de conservación se centra en facilitar esa interacción, establecer los criterios sobre el medio expositivo en función del estudio de la obra, documentar, comparar los resultados, etc.

En ocasiones, el museo no recibe la obra, sino que solo se le facilitan las instrucciones del artista, indicando las necesidades y requerimientos de la misma para poder llevar a cabo su plasmación material. Esta situación se da cuando la obra, montada, no puede ser conservada, necesita materiales nuevos, no es posible el traslado, debe adaptarse al espacio, etc. En estos casos, la conservación consiste en establecer las fases de trabajo, coordinar la labor de los diferentes profesionales que pueden verse involucrados, analizar y estudiar si los aspectos expositivos ya han sido definidos de manera permanente, es decir si son variables o no, velar por su conservación, etc. 


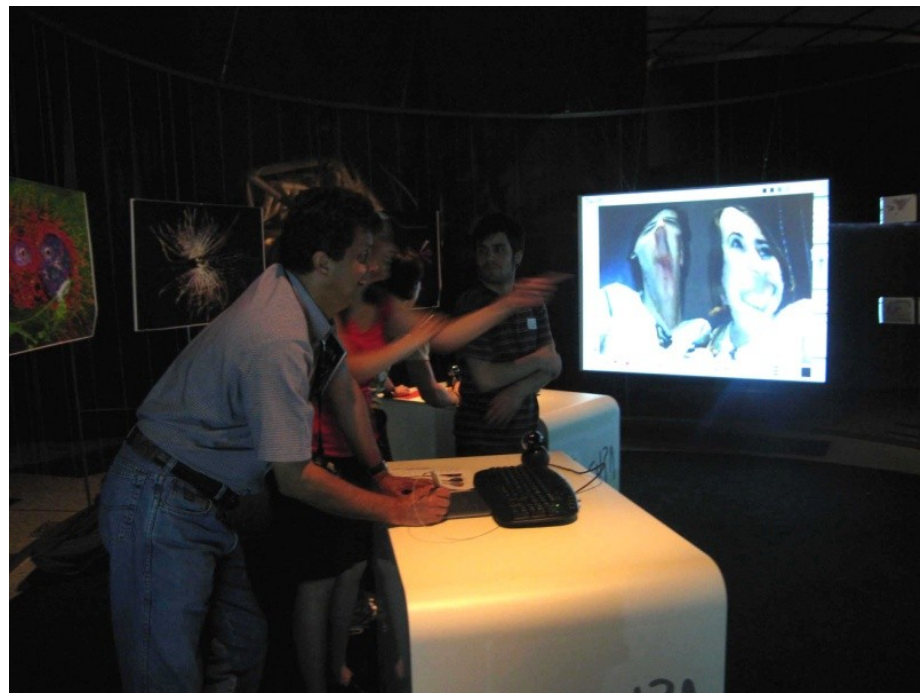

Figura 3. Participación del público en una obra interactiva

Es el ejemplo de la obra Circulation, del artista Hans Haacke, adquirida recientemente por el Museo Nacional Centro de Arte Reina Sofía. Esta obra está formada por un circuito de agua, que sigue un recorrido complejo a través de tubos conectados de manera específica. La adquisición de la obra se materializó inicialmente en un dossier con instrucciones, el Departamento de Conservación y Restauración puso en práctica una metodología adecuada a la obra, teniendo en cuenta todos los aspectos mencionados anteriormente.

En otros casos, el propio artista se involucra en el montaje de la obra, esto es importante, ya que los departamentos de conservación pueden así asesorar al artista en determinados aspectos de conservación, que no suelen tener en cuenta. Estos aspectos suelen ser válidos y útiles en sucesivos montajes.
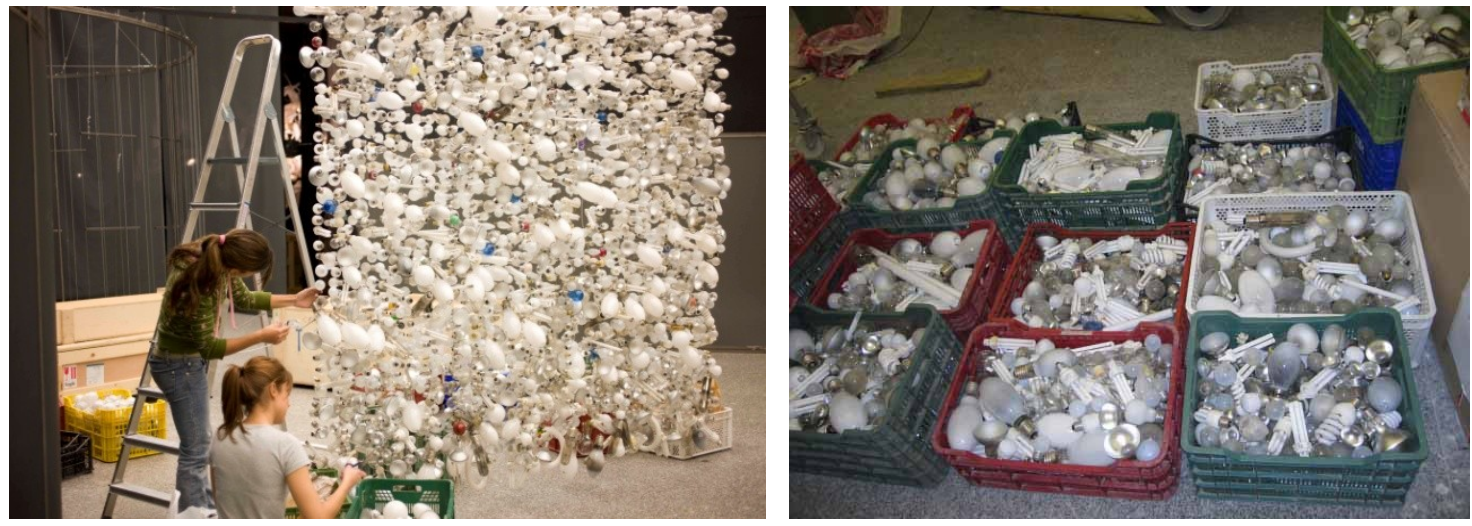

Figuras 4 y 5. Momento del montaje de la exposición Maquinas y Almas. Museo Nacional Centro de Arte Reina Sofía

La figura 4 muestra un momento del montaje de una de las obras de la exposición Máquinas y Almas, celebrada en el 2008 en el Museo Nacional Centro de Arte Reina Sofía. Para que la obra pudiera funcionar totalmente, necesitaba un sistema de luces externas y un sensor para que el público activara la obra, formando luces definidas por los movimientos del espectador, que se proyectaban en una pantalla formada por bombillas, el espacio debía estar en penumbra, para poder observar el juego de luces. En el caso de que el funcionamiento fuera incorrecto, o las 
características no estuvieran bien definidas, o hubiera interferencias con otras obras circundantes, se alteraría la obra y el espectador no podría apreciarla en su totalidad. En este caso además, era importante el material con el que se debía realizar la pantalla escultórica, ya que debía cumplir unos requisitos determinados: debía estar formada por bombillas fundidas de desecho, colocadas aleatoriamente.

Por cuestiones de conservación, esta obra debe ser montada en cada ocasión. El artista prestó la obra incluyendo un completo manual de instrucciones y requerimientos y colaboró en su montaje, siendo una fuente de información muy útil.

C) Obras de arte que pueden verse alteradas de manera puntual o permanente

El arte contemporáneo se modifica, a veces de manera voluntaria, forzada por el artista, o involuntariamente, por problemas expositivos o formales. Esto obliga a revisar y redefinir sus características.

Una obra puede ser creada para un lugar concreto, generando así determinadas necesidades y características que la definen y se estudian en ese momento. No obstante, un medio expositivo concreto no es necesariamente definitivo. En ocasiones, remodelaciones, exposiciones, préstamos u otras circunstancias, hacen que la obra se vea expuesta en diferentes entornos.

Como consecuencia de lo anterior, el espacio expositivo cambia, y con él se pueden perder ciertas características de la obra, aunque su identidad no varíe. La percepción y el medio de transmisión también corren el peligro de ser modificados, por lo que la labor de documentación es fundamental para evitar la pérdida de información de la obra. En este sentido, se definen los criterios para que ese nuevo espacio no distorsione la identidad de la obra.

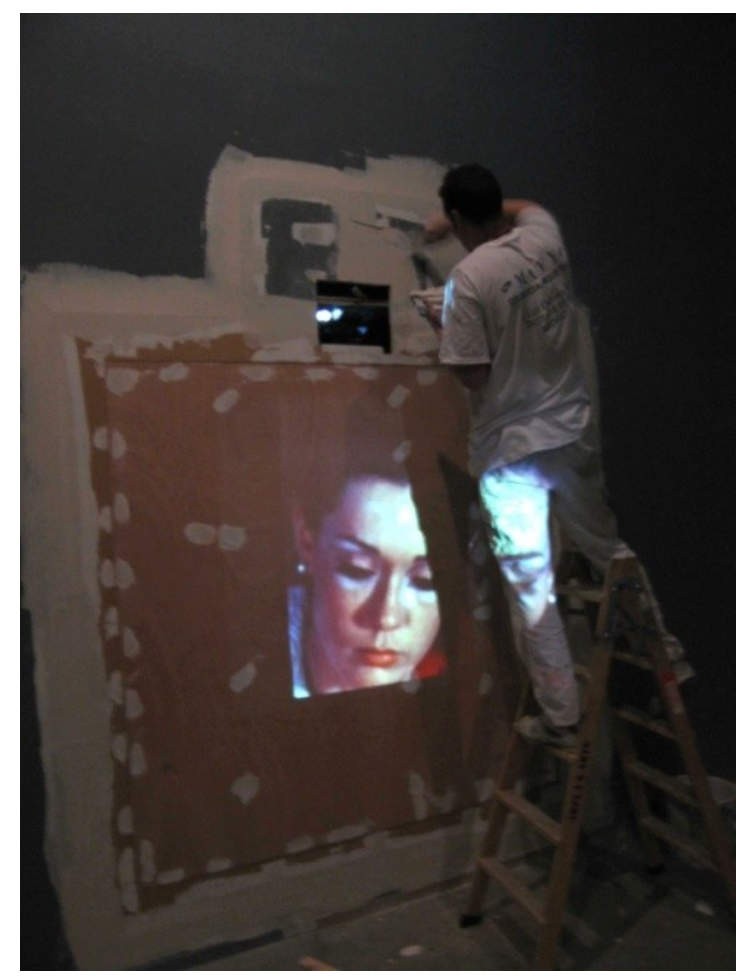

Figura 6. Montaje de una obra en una exposición temporal 
Las obras pueden plantear problemas en cuanto a los formatos y materiales que las conforman. Estos pueden quedar obsoletos en un momento determinado, es el caso del arte audiovisual, donde se usan diferentes formatos digitales y analógicos, que en ocasiones dificultan la durabilidad y estabilidad de la obra. En estos casos se establecen protocolos a nivel internacional y el restaurador aplica y define los criterios adecuados en cada momento. Por otro lado, la utilización de materiales orgánicos inestables (ciertas resinas, plásticos, materiales perecederos, etc.) obligan a crear protocolos de conservación e investigaciones exhaustivas, para evitar la degradación definitiva y la consecuente pérdida de la obra.

En ocasiones, los restauradores asesoran al artista cuando este, dadas las limitaciones de su obra (técnicas, expositivas, causadas por una pérdida de información que impide que el mensaje llegue al espectador...), decide revisarla de nuevo. En estos casos se asesora y coordina el trabajo para poder llegar a las soluciones y conclusiones necesarias.

D) Obras de arte fragmentarias o parciales

Son obras que tienen un medio y soporte específico en el momento de su creación, pero que pueden sufrir modificaciones y fragmentaciones, bien para ser exhibidas o conservadas, bien para que formen parte de otra obra o proceso artístico.

A veces, ciertas obras necesitan modificarse o fragmentarse en un soporte diferente. Las obras efímeras por ejemplo, para poder ser mostradas posteriormente, se pueden registrar en otros formatos, como el vídeo. Esto puede suponer un riesgo de pérdida de información (ya que la grabación puede ser parcial), por lo que la documentación exhaustiva durante todo el proceso y evolución de la obra y su transferencia es fundamental para conservarla.

Por otro lado, es importante analizar la obra completa para poder identificar esa fragmentación (a través del estudio e investigación), ya que puede tomarse por unitaria erróneamente.

Un ejemplo de esto es la obra de la performista Marina Abramovich, habituada a grabar y documentar fotográficamente todas sus actuaciones. Su obra, en muchas ocasiones, puede ser mostrada a través de fragmentos de esa documentación (vídeos o fotografías), además, esta documentación puede también formar parte de otras instalaciones, ampliando su significado en relación al nuevo entorno en el que se encuentra.

\section{La documentación dentro de la conservación del arte contemporáneo}

La labor de conservación aporta al estudio del arte contemporáneo documentación e información trascendente, tanto del artista como de la obra de arte, en todos sus ámbitos (conceptuales, formales, expositivos...). Para llevar a cabo una metodología apropiada, que sea capaz de generar y conservar esta documentación e información, se plantean diferentes fases:

- Planteamiento del problema. Este puede estar relacionado con el entorno expositivo, la restauración y/o conservación a largo o corto plazo, materialización de una propuesta del artista, recuperación de la pérdida de información de una obra por modificaciones, mutaciones, etc., todo ello en relación con las tipologías generales mencionadas anteriormente.

- Establecer las vías de búsqueda de las fuentes de información: estas pueden ser, el contacto directo con el artista, su entorno profesional (ayudantes, colaboradores...) o personal, el análisis de la obra (materiales, conceptuales...), los precedentes expositivos (características predeterminadas, riesgos de mutaciones...), el seguimiento de la obra durante su exhibición, etc.

- Recopilación, organización y almacenamiento de la información resultante. 
- Constitución de los parámetros de actuación consecuentes con la información obtenida.

- Organización y conservación de la documentación. Normalmente esta fase es compleja, ya que nos encontramos con material de diversa naturaleza (grabaciones, entrevistas registradas en audio, vídeo, conversación telefónica, e-mail, contactos directos, documentos escritos, fotografías, maquetas, croquis, dibujos, etc.). Esta pluralidad de soportes obliga a crear protocolos de almacenamiento específicos, donde se incluyen, entre otros, la realización de copias de seguridad o estandarización de formatos.

- Creación de bases de almacenamiento de datos y centros de documentación accesibles, según el tipo de documentación generada.

- Contrastar y analizar la información, previa y posterior a la intervención para extraer conclusiones. De ello depende, en muchas ocasiones, evitar la pérdida de información que genera interferencias o mutaciones sobre las obras de arte.

- Actualizar y revisar protocolos y documentación con relación a las obras.

\section{Conclusión}

La conservación se está convirtiendo en una fuente para el estudio del arte contemporáneo ya que su labor se implica en muchos procesos artísticos, que como ya hemos dicho, pueden condicionar la evolución de esa obra o artista. Los conservadores y restauradores de arte contemporáneo están realizando estudios e investigaciones para poder desarrollar cada una de estas cuestiones en profundidad.

Los departamentos de conservación de los museos y centros de arte contemporáneo son una referencia en este sentido, ya que sus instituciones generan y gestionan, hoy en día, gran parte del arte actual. Esto les proporciona conocimientos y datos de primera mano sobre los artistas y sus obras.

Toda la información generada debe ser analizada, catalogada y conservada, además esta debe ser accesible a investigadores e historiadores del arte. Por ello es fundamental la creación de protocolos de almacenamiento y conservación de la documentación. La colaboración entre los diferentes profesionales implicados en el estudio del arte contemporáneo es necesaria para poder realizar esta labor.

\section{Agradecimientos}

A todo el Departamento de Conservación y Restauración del Museo Nacional Centro de Arte Reina Sofía. 


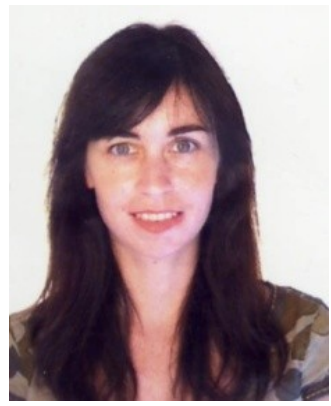

\author{
A. García González \\ Museo Nacional Centro de Arte Reina Sofía \\ alicia.restaura@gmail.com
}

Licenciada en Historia del Arte y Bellas Artes por la Universidad de Salamanca. Titulada en Conservación y Restauración por la E.S.C.R.B.C. de Madrid. DEA en "Teoría e Historia del Arte en la Edad Contemporánea", Universidad Complutense de Madrid.

Desde el 2008 colabora con el Departamento de Conservación del Museo Nacional Centro de Arte Reina Sofía gracias a una beca del Ministerio de Cultura. 